\title{
Ambient Noise Characterization of Shallow Water Environment
}

\author{
Tri Budi Santoso \\ Signal Laboratory, Electronic Engineering Polytechnic Institute of Surabaya \\ Jl Raya ITS, Sukolilo \\ E-mail: tribudi@pens.ac.id
}

\begin{abstract}
Understanding of environmental characteristic is a key factor in design of communication system. This paper describes an ambient noise characterization of measurement in the shallow water of Surabaya bay. The result showed that probability density function (pdf) of ambient noise is close to a Gaussian distribution with the mean of $-2.85 \times 10^{-5}$ and deviation standard of $9.87 \times 10^{-4}$. Validation has been done by using mean square error (MSE) and Bhattacharya distance. Fitting between cdf of measurement result and theory was done by using MSE with the value of and Kolmogorov Smirnov test with the value of $3.9 \times 10^{-4}$ and $4.9 \times 10^{-2}$ respectively. In the frequency domain analysis, it is showed that in the range of $0 \sim 9 \mathrm{kHz}$ has a deceasing level from -100 until $-140 \mathrm{~dB}$. In the frequency of $9 \sim 13.5$ $\mathrm{kHz}$, the spectral is flat as like a white. It can be used as a reference in decision of range frequency of underwater acoustic communication systems that will be applied.
\end{abstract}

Keywords: ambient noise, statistical characteristics, underwater acoustic.

\section{INTRODUCTION}

Understanding of statistical behavior characterization of additive noise and fading processes experienced by the transceiver is necessary to obtain basic performance limitations of underwater acoustic channel [1] and [2]. Ambient noise generally appears continually at a certain location. This can corrupt the signal emitted by the underwater acoustic equipment. So, the detection of background noise is important to get the signal-to-Noise ratio (SNR) of acoustical based underwater instrumentation.

Ambient noise determines the baseline sound scape, which in general is background noise that appears in a location at sea at certain times. Practically, the ambient noise is associated with noise, like the flow of water around the sonar. In the sonar system processing, background noise, has a similarity in the time, locations, and depth, where is the signal to be detected [3] and [4].

Research of wind effects on ambient noise has been carried out [5]-[9]. Generally, the research is related to the effects of wind and ship movement on acoustic noise that appeared. The discussion is focused on the observation 
of noise level and its relation to the wind speed, other effects of weather on noise, and other noise from sea traffic. But, these papers have no direct relation with performance of communication system, and do not describe statistical characterization of the noise.

A time-frequency analysis of ambient noise measurement has been presented [10] [12]. Measurement process has been carried out in the shallow water, with the $10 \mathrm{~m}$ depth, and time-frequency analysis has been conducted by using Fourier and Wavelet transforms. But, the discussion in this paper is not related to the objective of the characterization of ambient noise.

In this paper, we present measurements and characterization of ambient noise that has been conducted in coastal environments of Surabaya, which represent a condition of the tropical shallow water environment. Characterization has been done by off-line processing to obtain the noise properties in time and frequency domains. Time domain analysis is focused on probability distributions, and validated by using mean-square-error (MSE) and Bhattacharya distance. Frequency domain analysis can reveal the degree of whiteness of ambient noise in tropical shallow water. Our result shows that in the low frequency band up to around $8 \mathrm{kHz}$ the ambient noise power spectrum approaches a negative logarithmic shape. However, at frequencies above $8 \mathrm{kHz}$, the noise appears to be white. It is very important in communication system design and analysis.

The paper is organized as follows. Section 2 describes the sea state and the set-up of our experiment. In section 3, experimental result is analyzed in time and frequency domains. Section 4 summarizes the paper with some concluding.

\section{EXPERIMENTAL SET-UP}

\subsection{Description of Environmental Measurement}

Measurement has been conducted in Surabaya bay, with taking data from some location in $\left(7^{\circ} 0^{\prime} \sim 7^{\circ} 30^{\prime}(\mathrm{S}) ; 112^{\circ} 30^{\prime} \sim 113^{\circ} 0^{\prime}(\mathrm{E})\right)$ coordinate. This position is expected to be able to represent the shallow water environment with relatively busy traffic, because it is close to traffic lines between Surabaya - east of Indonesia. Noise measurement position is at a $2.4 \mathrm{~m} \mathrm{depth}$, and is located at about $200 \mathrm{~m}$ from the beach, as in Figure 1.

The sea conditions at the measurement time with the wind slowly toward the coast, relatively calm water surface, water flow leads to the beach with a small splash around $0 \sim 10 \mathrm{~cm}$. This represented a measurement condition with a sea state of 1 [12]. Environment temperature was about $32.7^{\circ} \mathrm{C}$, and aerial noise of $46.8 \sim 53 \mathrm{~dB}$. 


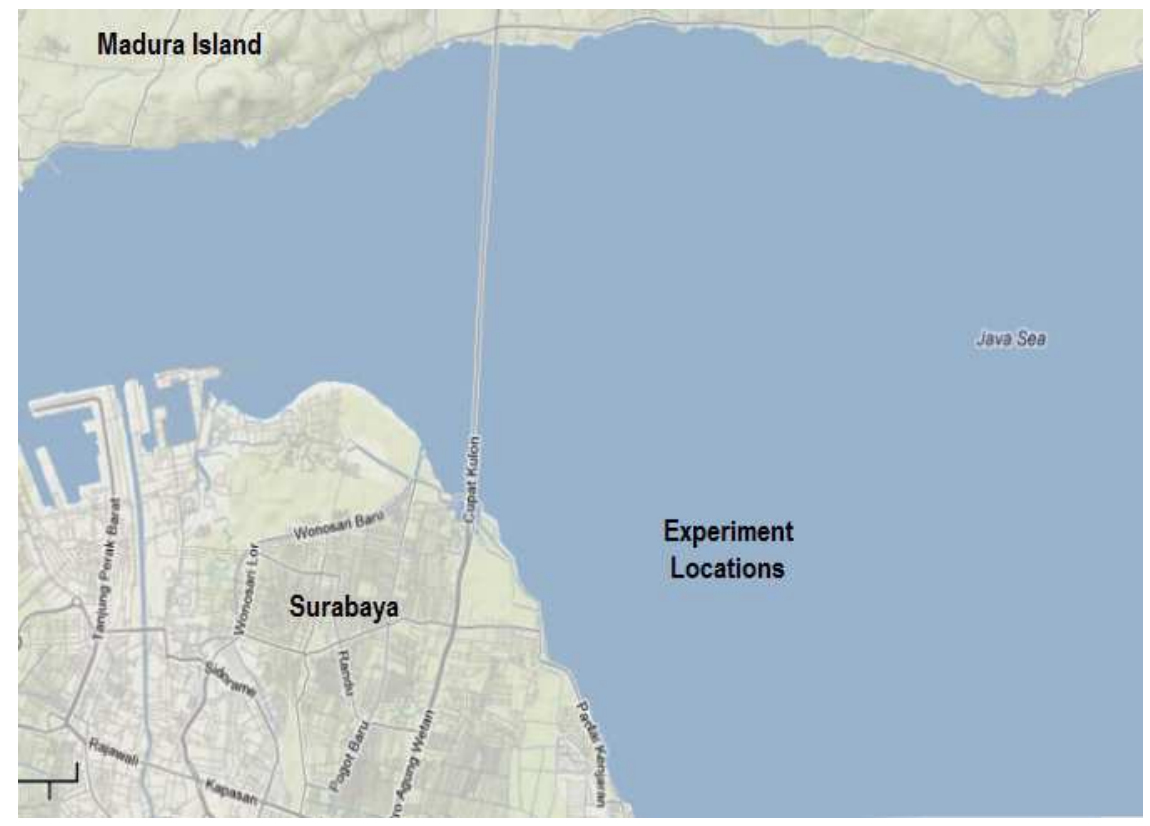

Figure 1. Location of ambient noise measurement

\subsection{Hydrophone Normalization}

The objection of the normalization process is to determine the sensitivity of hydrophone to be used. This is done to anticipate a difference in sensitivity among the hydrophones [13] and [14]. Normalization process has been conducted with a setup as Figure 2.

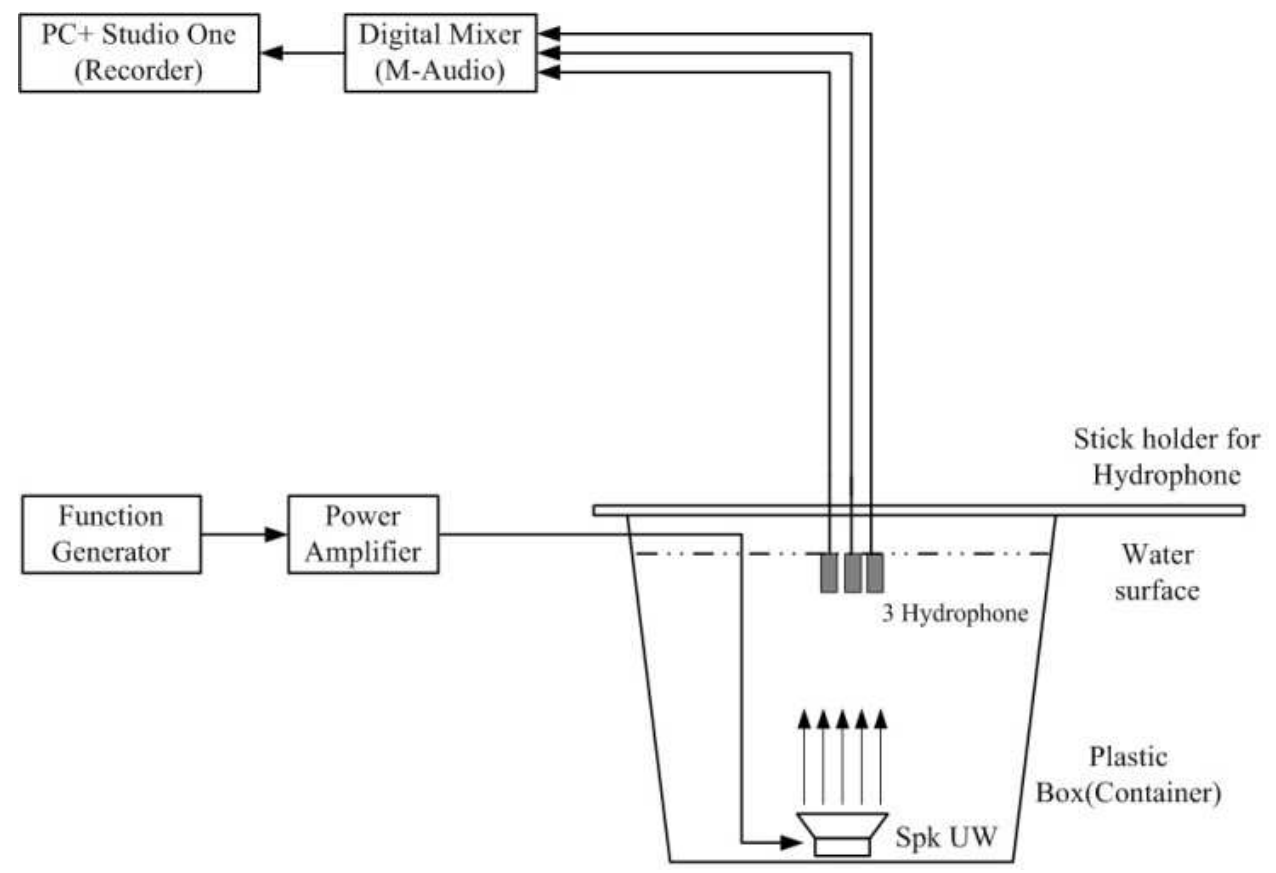

Figure 2. Setup of Hydrophone normalization 
The sound source is placed at the bottom on the box, with the speaker facing up. Hydrophones position on the surface of the water, and facing to sound source. With this position, the sound propagates directly from the bottom to the surface without any reflections.

Testing is done by using linear frequency modulation (LFM) signals, with a frequency sweep $1 \sim 15 \mathrm{kHz}$, generated from a PC. Processing gain is obtained by using audio amplifier, converted to an acoustic signal with underwater speaker. An acoustic signal is captured by 3 hydrophones, and converted back to an electric signal. Gain of digital mixer (M-Audio) is set of $10 \mathrm{~dB}$, and saving process by software without additional gain.

Signal in each frame is transformed into the frequency domain by utilizing the periodogram function, which gives an overview of the estimated power spectral density (PSD). If $x(t)$ is a discrete time signal $\{x(t) ; t=0, \pm 1, \pm 2, \ldots\}$ is assumed to be a sequence of random variables. The covariance of $x(t)$ is defined as:

$$
r(k)=E\left\{x(t) x^{*}(t-k)\right\}
$$

Where, $E\{\cdot\}$ denotes the expectation operator which averages over the ensemble of realization. The value of $x(t)$ is assumed to depend only on the lag between the two samples averaged. Variable $x^{*}(t-k)$ is the complex conjugate of $x(t)$ with shift version. The PSD is defined as discrete time Fourier transform (DTFT) of the covariance sequence

$$
\phi(\omega)=\sum_{k=-\infty}^{\infty} r(k) e^{-j \omega k}
$$

The periodogram output of all the frames averaged to represent one of the channel responses of M-Audio equipment.

Channel 1 and Channel 2 have similar sensitivity, so the reception ratio, in the frequency range of $1 \sim 12 \mathrm{kHz}$ is about 1.2 . But, at a frequency of $14 \mathrm{kHz}$ Channel 2 tends to 2.3 times more sensitive than Channel 1. Channel 4 also has similar sensitivity with Channel 1 . Channel 3 is more sensitive compare to the other channels, comparing with Channel is twice more sensitive at the frequency of $1 \mathrm{kHz}$, and almost 2.5 times more sensitive at higher frequency. It can be shown on the top part of the chart. Channel 3 is also more sensitive than the channel 2, so that the ratio of Channel 2 to Channel 3 is about 0.5. Comparison of Channel 4 to Channel 3 also describes the same thing with the channel 2, which shows the sensitivity of 0.5 compared to Channel 3. Picture comparison sensitivity is a reference in the compensation transform and normalized the data input on a different channel in the M-Audio.

\subsection{Experimental Setup}

Environmental noise measurements have been carried out by conditioning the ship's engine turned off, with minimum activity to avoid the appearance of unwanted noise. Rack mounted and hydrophones cables have 
been wrapped in to reduce the influence of a collision with the flow of sea water. Experimental setup is as Figure 3.

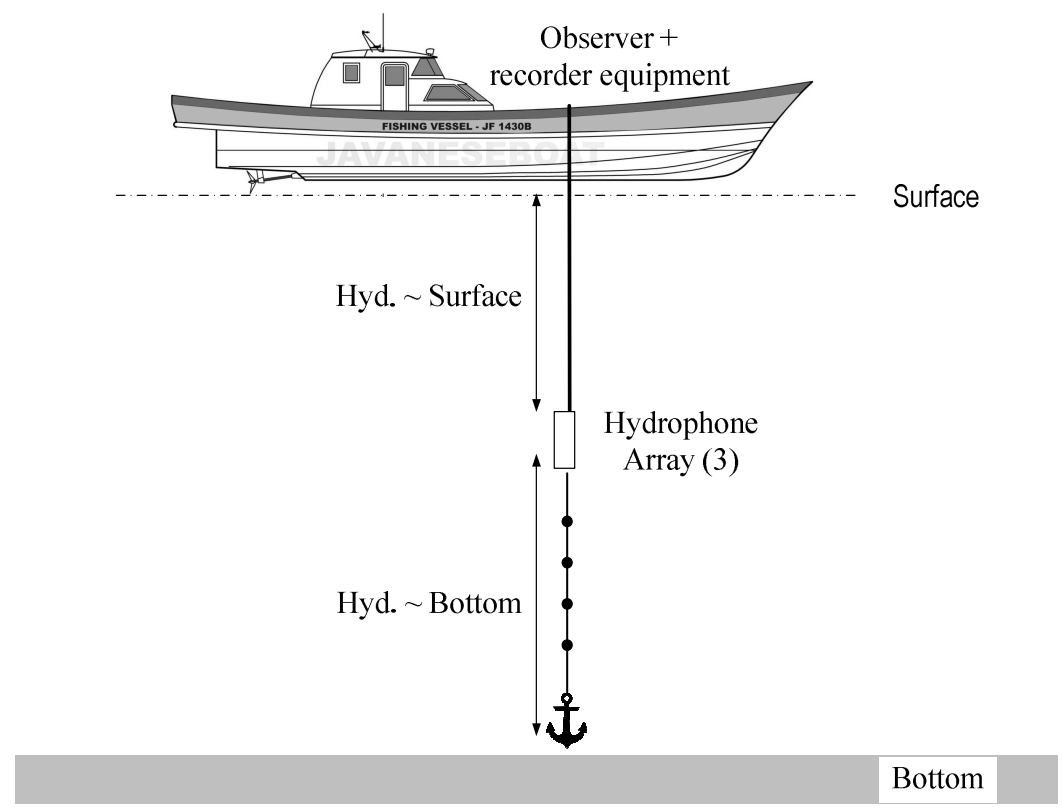

Figure 3. Set Up of ambient noise measurement

The recording has been carried out in two different locations, each performed three times, with the duration of 20 second for each recording. Thus, totally obtaining 18 data measurements.

The hydrophones have following specifications: frequency range of $1 \mathrm{~Hz} \sim$ $100 \mathrm{kHz}$, sensitivity-190 dB relative to $1 \mathrm{~V} / \mu \mathrm{Pa}( \pm 4 \mathrm{~dB} 20 \mathrm{~Hz} \sim 4 \mathrm{kHz})$, with an internal capacitance of $25 n \mathrm{~F}$. The radiation pattern of capturing signal is almost omnidirectional, with the strongest response at $0^{\circ}$, or perpendicular to hydrophone surface. The response decreases for signal with arriving an angle of $\geq 10^{\circ}$.

Data recording of the ambient noise is statistically characterized to obtain a signal levels histogram. The fitting process of the resulting pdf with standard distribution functions such as Gaussian is done by using MSE and Bhattacharya distance.

\section{EXPERIMENTAL RESULT}

\subsection{Time Domain Analysis of Ambient Noise}

Overview of ambient noise in time domain provides information about noise level fluctuations as a function of time. Most of the noise level has a low value around the zero level, and any part that has a value of about $-0.01 \mathrm{~V}$ and $0.01 \mathrm{~V}$. Typically, the picture of noise level in the time domain is shown in Figure 4.

The two measurement data that obtained from two different times are treated as groups $\mathrm{X}$ and $\mathrm{Y}$. The elements in the first group are $x_{1}, x_{2}, \ldots . x_{n} \sim$ 
$N\left(\mu_{\mathrm{x}}, \sigma^{2}\right)$, and the elements in the second group are $y_{1}, y_{2}, \ldots y_{\mathrm{n}} \sim N\left(\mu_{\mathrm{y}}, \sigma^{2}\right)$. The overall mean or combined mean is the sum of all the observations divided by the total number of observations $2 \mathrm{n}$. Because each group has equally many observations, the overall mean is just the midpoint of the two means.

$$
\mu_{\mathrm{c}}=\left(\mathrm{n} \mu_{\mathrm{x}}+\mathrm{n} \mu_{\mathrm{x}}\right) /(\mathrm{n}+\mathrm{n})=\left(\mu_{\mathrm{x}}+\mu_{\mathrm{x}}\right) / 2
$$

The combined variance follows the same structure, but we have to sum across all of the X observations and all of the Y observations.

$$
\sigma_{c}^{2}=\frac{\left(\sum_{i=1}^{n}\left(x_{i}-\bar{x}\right)^{2}+\sum_{i=1}^{n}\left(y_{i}-\bar{y}\right)^{2}\right)}{2 n-1}
$$

The average of deviation standard is a square root of combined variance. With this statistical approach, it has been obtained a description that ambient noise has an average value of $-2.85 \times 10^{-5} \mathrm{~V}$, and standard deviation of $9.87 \mathrm{x}$ $10^{-4} \mathrm{~V}$.

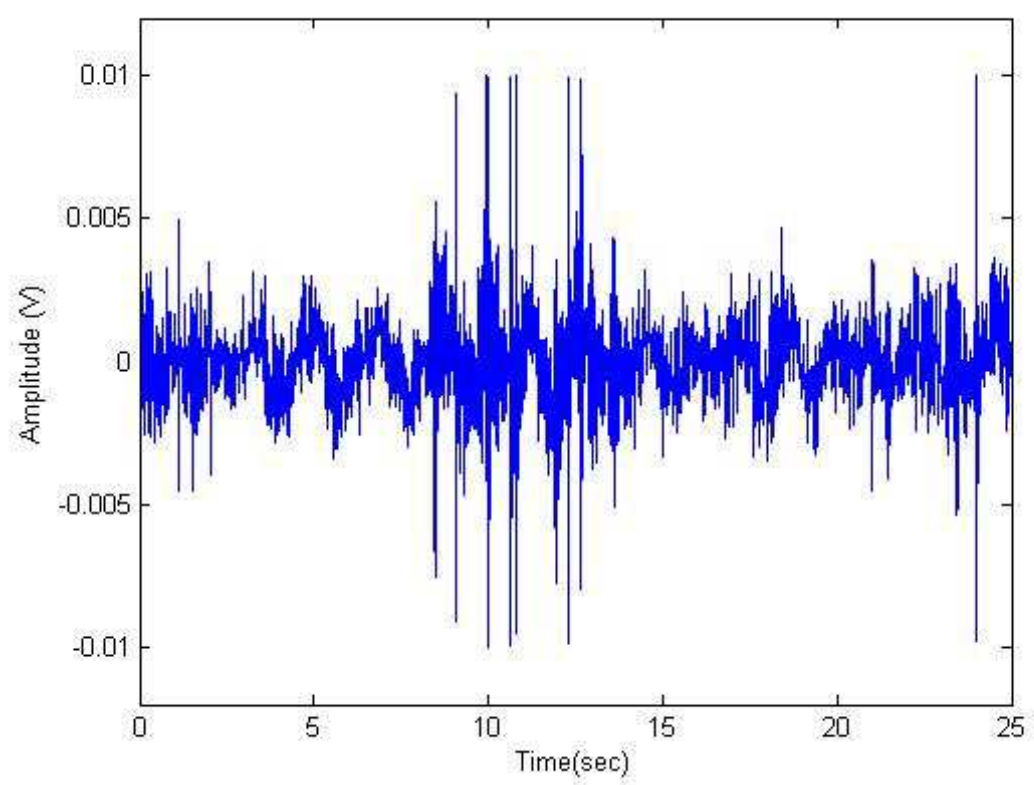

Figure 4. Measurement result of ambient noise

Data outputs of noise level of measurement results, is converted to histogram form, to obtain information about distribution pattern spreading in pdf format. By averaging all pdf from measurements using three hydrophones, we obtained a description of the distribution pattern as shown in Figure 5.

The fitting process is done in the form of a pdf by using MSE and Bhattacharya distance. MSE is a fitting calculation with simple techniques and has been widely used in statistical analysis. Bhattacharya distance is widely used in the determination of the size of the closeness between the two 
histograms of the signal level and pattern recognition. This technique is very simple and not affected by the size of the data, but as function of probability density function of the data [15].

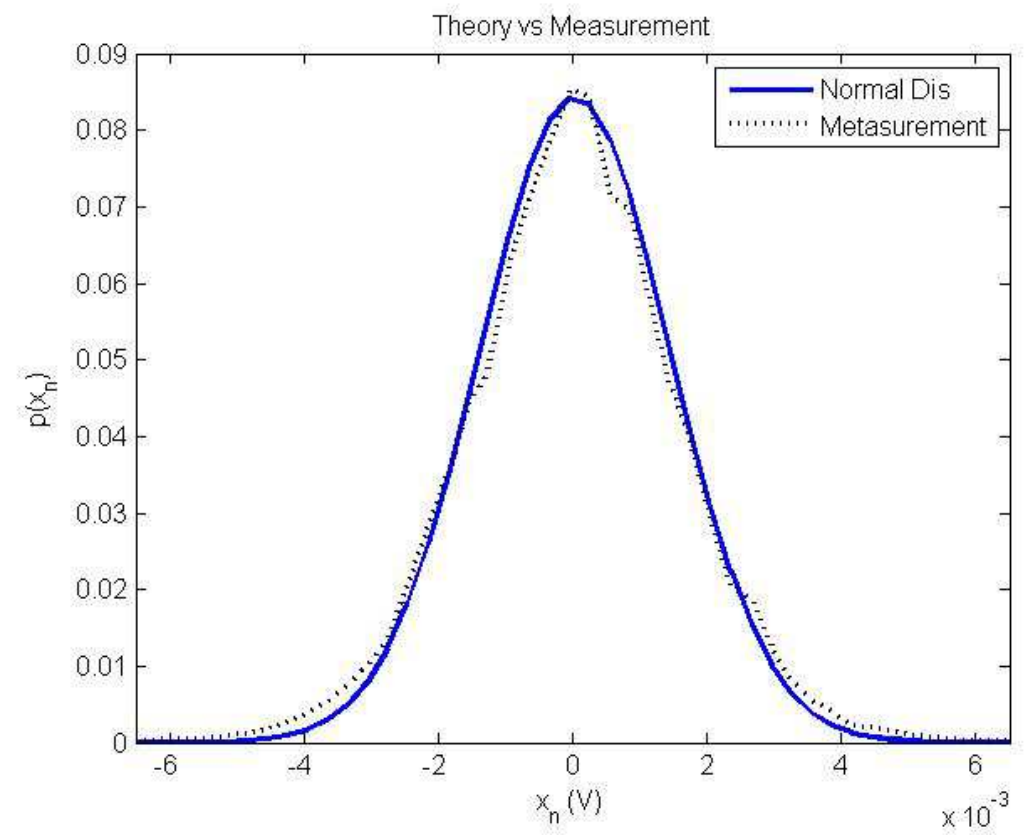

Figure 5. Pdf of ambient noise

If $p\left(x_{\mathrm{n}}\right)$ is a pdf of measurement data, and $q\left(x_{\mathrm{n}}\right)$ is pdf of standard distribution function, then the distance between them is defined as:

$$
B(P, Q)=-\ln \left(\int \sqrt{p\left(x_{n}\right) q\left(x_{n}\right)} d x_{n}\right)=-\ln (\rho)
$$

where

$$
\rho(P, Q)=\int \sqrt{p\left(x_{n}\right) q\left(x_{n}\right)} d x_{n}
$$

is known as Bhattacharya coefficient.

Fitting process with a pdf of Gaussian distribution function with zero mean, and standard deviation of $\sigma=9.87 \times 10^{-4}$, are: MSE $=9.96 \times 10^{-5}$ and Bhattacharya distance $=3.54 \times 10^{-4}$. This score is very small, and represent very a condition that measurement result is very close with a pdf of Gaussian distribution function.

Statistical analysis of the measurement data can be developed by using the cumulative distribution function (cdf). A technique for fitting between two cdf can be done by use Kolmogorov Smirnov test (KS-test). The test is initialized by data processing to obtain a cdf of $\mathrm{S}(\mathrm{x})$. We set $\mathrm{F}^{*}(\mathrm{x})$ as a hypothesis of standard cdf. The T variable is set as a statistic test to obtain the largest value, sup (suprenum) of vertical distance between $\mathrm{S}(\mathrm{x})$ and $\mathrm{F}^{*}(\mathrm{x})$. And, the value of $\mathrm{KS}$-test is expressed as:

$$
T=\sup \left|F^{*}(x)-S(x)\right|
$$


Cdf value is estimated by using the pdf output of measurement data. The cdf of ambient noise of measurement give an output as Figure 6 with the line'-o-'. Cdf of Gaussian function as comparator is generated by using the value of mean and variance of measurement data with the solid line. From these figures showed that between the measurement results and cdf theory has a similarity. The fitting process between cdf theory with measurement result and theory was done by using MSE and KS-test with the different value of $3.9 \times 10^{-4}$ and $4.9 \times 10^{-2}$ respectively. These values indicated that the cfd obtained from measurement has a high similarity with the theory.

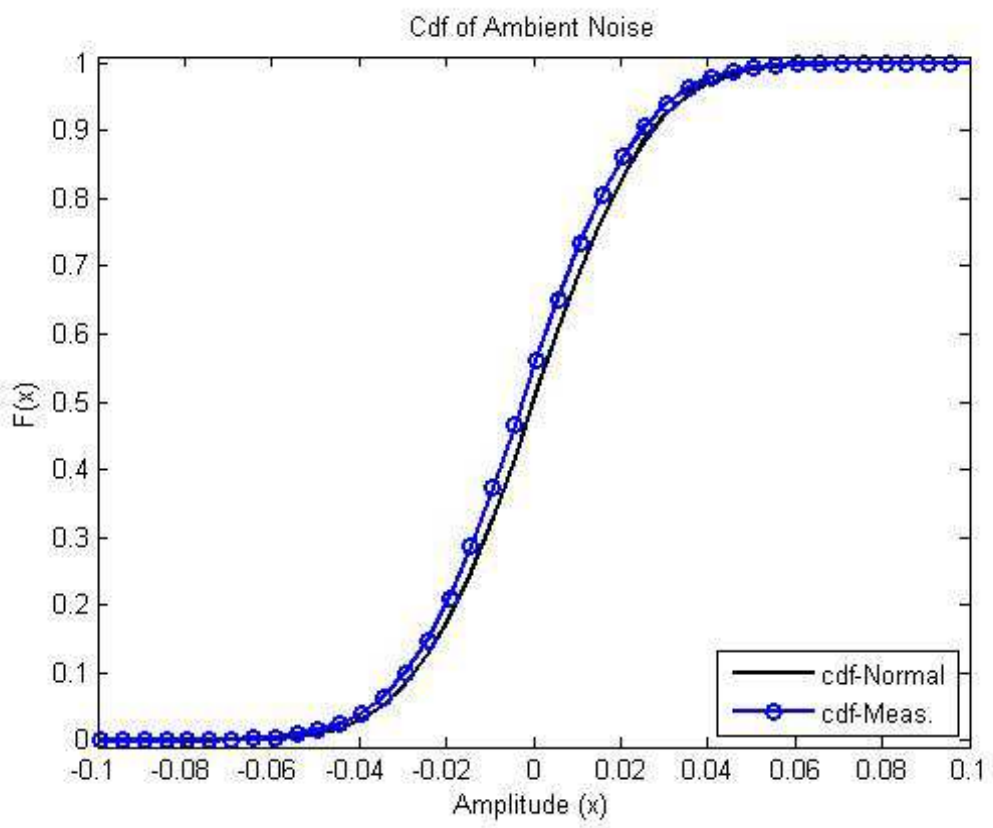

Figure 6. Cdf of ambient noise

\subsection{Frequency Domain Analysis of Ambient Noise}

Observations of the spectral power density give an illustration that the level of noise power as a function of frequency is not totally flat. In general, the results of ambient noise measurement give an overview spectrum shape as shown in Figure 7.

Ambient noise with frequency range of $0 \sim 9 \mathrm{kHz}$ has a trend of decreasing power level as a function of frequency, start from $-90 \mathrm{~dB}$ to -140 $\mathrm{dB}$. In the frequency range of $9 \sim 13.5 \mathrm{kHz}$, the noise power level is quite flat. In the frequency range of $14 \mathrm{kHz} \sim 18 \mathrm{kHz}$ the noise power level has a fluctuation. With averaging of all the measurements provide a picture noise spectrum is relatively flat in some specific range frequency.

From the frequency domain analysis of the ambient noise measurements we can see that frequency range of $9 \sim 13.5 \mathrm{kHz}$ has a flat spectrum and indicate whiteness of the noise in that spectral. The result indicates that in that frequency range, the channel has a white Gaussian noise. Thus we can 
adopt the model of digital communication system that's already widely used in wireless system design.

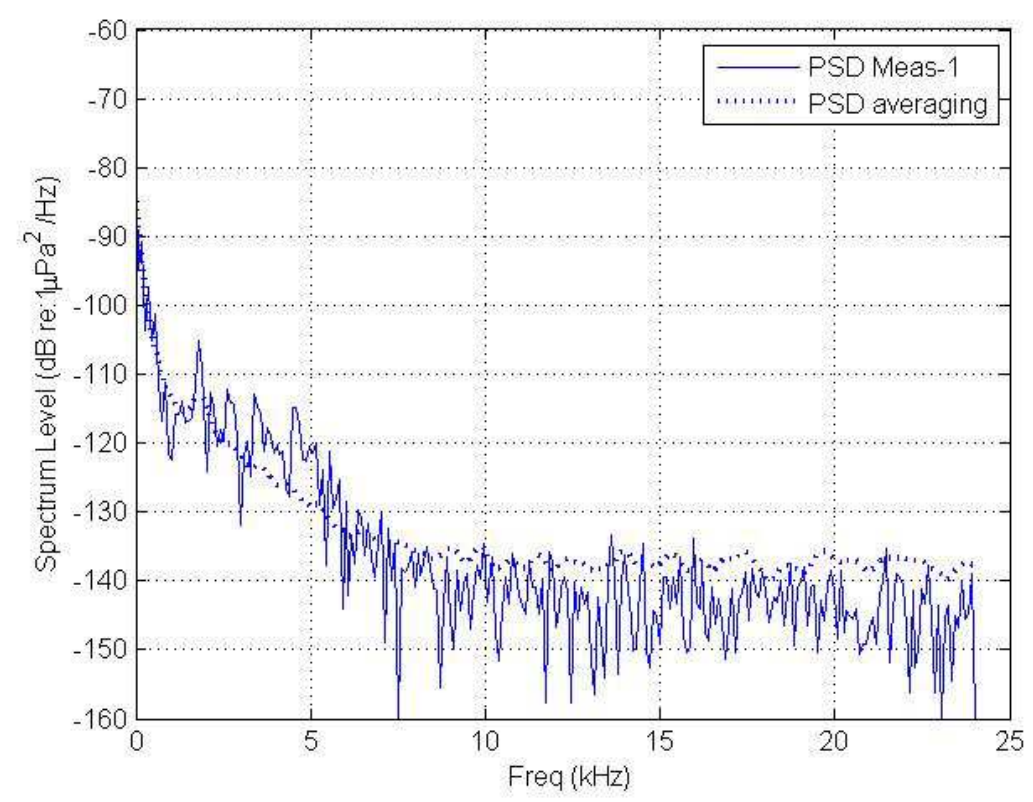

Figure7. Spectrum of ambient noise

\section{CONCLUSION}

This paper has presented an ambient noise characterization from measurement in the shallow water of Surabaya bay. From the analysis, it is shown that the pdf of ambient noise is close to the nature of a Gaussian distribution with zero mean and standard deviation of $9.87 \times 10^{-4} \mathrm{~V}$. In the frequency range of $9 \mathrm{kHz} \sim 13.5 \mathrm{kHz}$, it shows a flat spectrum which represents an AWGN channel condition. It can be used as a ref that the modeling of the UWA communication system can be done by adopting the model of digital communication systems in AWGN channel, in the frequency range.

\section{Acknowledgements}

This work was supported by the Penelitian Fundamental, the fiscal year 2015, from the Indonesian Ministry of Research, Technology and Higher Education. We would like to thank Signal Processing Laboratory, which has given support to carry out the measurements.

\section{REFERENCES}

1. M. Stojanovic, “Underwater Acoustic Communications: Design Considerations on the Physical Layer", proceeding of Fifth Annual Conference on Wireless on Demand Network Systems and Services, 2008. WONS 2008. 
2. M. Badiey, Y. Mu, J.A. Simmen, and S.E. Forsthe, "Signal Variability in Shallow-Water Sound Channels", IEEE Journal of Oceanic Engineering, Vol.25, no.4, October 2000.

3. P. C. Etter, "Advanced Applications for Underwater Acoustic Modeling", Hindawi Publishing Corporation, Advances in Acoustics and Vibration, Volume 2012, Article ID 214839.

4. Poikonen, and S. Madekivi, "Recent Hydro Acoustic Measurement and Studies in the Gulf of Finland", Proceedings of the International Conference "Underwater Acoustic Measurements: Technologies \&Results", Heraklion, Crete, Greece, 28 $8^{\text {th }}$ June - 1st, July 2005.

5. M. Ashokana, G. Latha, and R. Ramesh, "Analysis of Shallow Water Ambient Noise Due to Rain and Derivation Rain Parameters", Elsevier, Applied Acoustic, Vol. 88 (2015), pp: 114-122

6. S. Najeem, M.C. Sanjana, G. Latha, and P.E.Durai, "Wind Induced Ambient Noise Modeling and Comparison with Field Measurements in Arabian Sea", Elsevier, Applied Acoustics, Vol. 89 (2015), pp: 101-106.

7. S.S. Murugan,V. Natarajan, and R.R. Kumar, "Noise Model Analysis and Estimation of Effect due to Wind Driven Ambient Noise in Shallow Water", Hindawi Publishing Corp., International Journal of Oceanography, Vol. 2011, Article ID 950838.

8. M. M. Mahanthy, M. C. Sanjaya, G. Latha, and G. Raguraman, “An Ivestigation on The Fluctuation and Variability of Ambient Noise in Shallow water of South West Bay of Bengal", Indian Journal of Geo Marine Science, Vol.43 (5), Mat 2014, pp.747-753.

9. Arnab Das, "Shallow Ambient Noise Variability due to Distant Shipping Noise and Tide", Elsevier, Applied Acoustics, Vol. 72 (2011), pp: 660-664.

10. R.K. Shastri, Y.V. Joshi, and A. Das, "Time Frequency Analysis of Underwater Ambient Noise in Tropical Littoral Waters", IEEE OCEANSan Diego, 23-27 September 2013.

11. J.R. Short, "High-Frequency Ambient Noise and Its Impact on Underwater Tracking Ranges", IEEE Journal of Oceanic Engineering, Vol. 30, No. 2, April 2005.

12. G.B. Deane and M.J. Buckingham, "Vertical Coherence of Ambient Noise in Shallow Water Overlying a Fluid Seabed", J. Acoustic Society of America, December 1997, Vol.102, No. 6, pp: 3413-3424.

13. A.E. Isaev, Chen Yi, A.N. Mateev, and Ping Zihong, “COONET.AUV.W-S1 supplementary comparison of free-field hydrophone calibration in the Frequency range of $250 \mathrm{~Hz}$ to $8 \mathrm{kHz}$ ", Metrologia, Vol. 52, 2015, doi: 10.1088/0026-1394/52/1A/09001.

14. D. Tom Dakin, Jeff Bosma, John Dorocicz, and Nicolai Bailly, “Calibrating Low Frequency Digital Hydrophones", International Conference and Exhibition on Underwater Acoustics (UA2013), Corfu, Greece, 23-26 June, 2013. 
15. S.H. Cha, "Taxonomy of Nominal Type Histogram Distance Measures", American Conference on applied Mathematics (Math'08), Harvard, Massachusetts, USA, March 24-26, 2008. 